\title{
1 Emergence of the subapical domain is associated with the midblastula
}

\section{2 transition}

3 Anja Schmidt (2), Jörg Großhans (1,2)

4 (1) Professur für Entwicklungsgenetik, Fachbereich Biologie, Philipps-Universität

5 Marburg, Karl-von-Frisch-Straße 8, 35043 Marburg, Germany

6 (2) Institut für Entwicklungsbiochemie, Universitätsmedizin, Georg-August-

7 Universität Göttingen, Justus-von-Liebig-Weg 11, 37077 Göttingen, Germany

8 Key words: cortical domains, epithelial domains, subapical, Canoe, midblastula

9 transition, zygotic genome activation

\section{Abstract}

11 Epithelial domains and cell polarity are determined by polarity proteins which are

12 associated with the cell cortex in a spatially restricted pattern. Early Drosophila

13 embryos are characterized by a stereotypic dynamic and de novo formation of cortical

14 domains. For example, the subapical domain emerges at the transition from syncytial

15 to cellular development during the first few minutes of interphase 14 . The dynamics

16 in cortical patterning is revealed by the subapical markers Canoe/Afadin and ELMO-

17 Sponge, which widely distributed in interphase 13 but subapically restricted in

18 interphase 14. The factors and mechanism determining the timing for the emergence

19 of the subapical domain have been unknown. In this study, we show, that the restricted

20 localization of subapical markers depends on the onset of zygotic gene expression. In

21 contrast to cell cycle remodeling, the emergence of the subapical domain does not

22 depend on the nucleo-cytoplasmic ratio. Thus, we define cortical dynamics and 
23 specifically the emergence of the subapical domain as a feature of the midblastula

24 transition.

\section{Author summary}

26 Midblastula transition is a paradigm of a developmental transition. Multiple processes

27 such as cell cycle, cell mobility, onset of zygotic gene expression, degradation of

28 maternal RNA and chromatin structure are coordinated to lead to defined changes in

29 visible morphology. The midblastula transition in Drosophila embryos is associated

30 with a change from fast nuclear cycles to a cell cycle mode with gap phase and slow

31 replication, a strong increase in zygotic transcription and cellularization. The timing

32 of the processes associated with the midblastula transition are controlled by the onset

33 of zygotic gene expression or the nucleocytoplasmic ratio. Here we define the

34 patterning of cortical domains, i. e. the emergence of a subapical domain as a novel

35 feature of the midblastula transition whose appearance is controlled by the onset of

36 zygotic transcription but not the nucleocytoplasmic ratio. Our findings will help to

37 gain further understanding of the coordination of complex developmental processes

38 during the midblastula transition.

\section{Introduction}

40 The cell cortex underlies the plasma membrane and consists of a layer of F-actin and

41 associated proteins, including actin nucleators, regulators and myosin motors.

42 Proteins, such as ERM proteins, link F-actin to the plasma membrane (1). Typical for

43 epithelial cells are cortical domains, which contain marker proteins specific for the

44 respective domain in addition to the general set of cortical proteins. For example, Par-

45 3/Bazooka (Baz) typically marks the subapical domain, whereas Par-1 marks the

46 lateral domain (2,3). Although mutual exclusion of such marker proteins has been

47 shown to maintain boundaries between two domains in some cells, the mechanism for 
48 initial establishment of the domains and pattern formation is not well defined. The de

49 novo appearance of the first epithelium during cellularization in Drosophila embryos,

50 provides an excellent model to study the initial formation of cortical domains and

51 epithelial polarization (4).

52 Following a syncytial phase of development with rapid nuclear cycles typical for

53 insects, the first epithelium forms after about two hours of embryonic development as

54 a morphologically obvious feature marking the transitions from syncytial to cellular

55 blastoderm (5-7). This morphological change, often referred to as midblastula

56 transition (MBT) is associated with several cellular processes that appear to be

57 coordinated, including remodeling of the cell cycle, transition to a slow mode of DNA

58 replication, heterochromatin formation, ingression of the cellularization furrow,

59 elongation of the nuclei, and importantly activation of the zygotic genome $(6,8,9)$.

60 Concerning epithelial polarization it is important to note that the number of cortical

61 domains increases during the transition from two cortical domains (caps and intercaps)

62 in interphase $13(10,11)$ and three domains (apical, lateral, basal) during mitosis (12)

63 to the typical four domains. A dedicated subapical region positioned between the

64 apical and lateral domains emerges for the first time in development in interphase 14

$65(3,8)$.

66 It is unknown, if and how the emergence of the subapical domains is linked or

67 coordinated with the other processes associated with the midblastula transition. It has

68 been previously shown that zygotic transcription initiates the cell cycle remodeling

69 and is required for cellularization (13). The changes are due to specific zygotic genes,

70 e. g. slam, nullo, frs or to global signals such as transcription associated DNA

71 replication stress and DNA checkpoint activation (13). The emergence of the subapical

72 domain has not been investigated in this context, so far. 
73 The earliest marker proteins for the prospective subapical domain during onset of

74 cellularization are Canoe (Cno, Afadin in vertebrates) and the unconventional GEF

75 complex ELMO- Sponge $(8,14)$, which act upstream of Canoe possibly via control of

76 the small GTPase Rap1. Both Canoe and ELMO-Sponge are widely distributed during

77 the syncytial interphases and mitoses (nuclear cycles 10-13). Canoe is detected in cap

78 and intercap regions, whereas the ELMO-Sponge complex marks the actin caps and

79 control their formation (8). This disc-like pattern in pre-MBT interphases changes to

80 a ring-like pattern in interphase 14, when ELMO-Sponge initiate restriction of Canoe

81 to the prospective subapical region. Only during the course of cellularization, the

82 typical subapical proteins Bazooka/Par-3 and Armadillo (Arm, $\beta$-Catenin in

83 vertebrates) are enriched in the subapical region (15-17).

84 In this study, we investigate the role of zygotic gene expression and cell cycle

85 remodeling for the formation of the subapical domain. As Bazooka feeds back on

86 subapical restriction of Canoe later in cellularization, we tested the function of this

87 genetic interaction for the initial emergence of the subapical region. We show that the

88 localization of early subapical domain markers like ELMO-Sponge and Canoe

89 depends on onset of zygotic expression but not cell cycle remodeling and not on

90 bazooka during early cellularization.

\section{Results}

\section{Change of Canoe distribution pattern at the onset of interphase 14}

93 The subapical cortical domain emerges during the transition from syncytial to cellular

94 blastoderm for the first time during embryonic development. During this process the

95 localization pattern of the actin binding protein Canoe changes from a dispersed

96 pattern at the actin caps to a coalesced pattern at the prospective subapical domain

97 within about five minutes of the onset of cellularization in interphase 14 (Figure 1A) 
98 (8). Subapical restriction of Canoe depends on the small GTPase Rap1 and the

99 unconventional guanyl nucleotide complex ELMO-Sponge, which undergoes a

100 relocalization from discs in interphase 13 to rings in interphase 14 (8). New

101 cellularization furrows form between the daughter nuclei. After reached longest

102 extension in metaphase, these furrows gradually retract in the second half of mitosis

103 to a length of about $3 \mu \mathrm{m}(32,33)$ (Figure 1B). We applied our live imaging assay with

104 embryos expressing the subapical marker CanoeYFP and basal marker CherrySlam to

105 reveal the kinetics of marker segregation. Axial stacks were recorded and

106 computationally projected to sagittal sections. During mitosis, Cherry Slam was

107 detected at the tip of the metaphase furrow, whereas CanoeYFP was spread along the

108 full length (Figure 1C). It is important to note the difference between "old"

109 cellularization furrows, which arise from retracting metaphase furrows, and "new"

110 cellularization furrows, which ingress between daughter nuclei. In "new"

111 cellularization furrows CanoeYFP associates within minutes to the in folding

112 membrane. In contrast, Canoe distribution is becomes subapically restricted at "old"

113 furrows starting from a wide distribution along the furrow (Figure 1C). CherrySlam

114 remains at the tip of "old" furrows, and gradually appears at the tip of "new" furrows

115 (Figure 1C) (8). Although we and others have uncovered the mechanism for subapical

116 restriction of Canoe $(3,8,15,17,34)$, the factors determining the timing have not be

117 studied. 
A

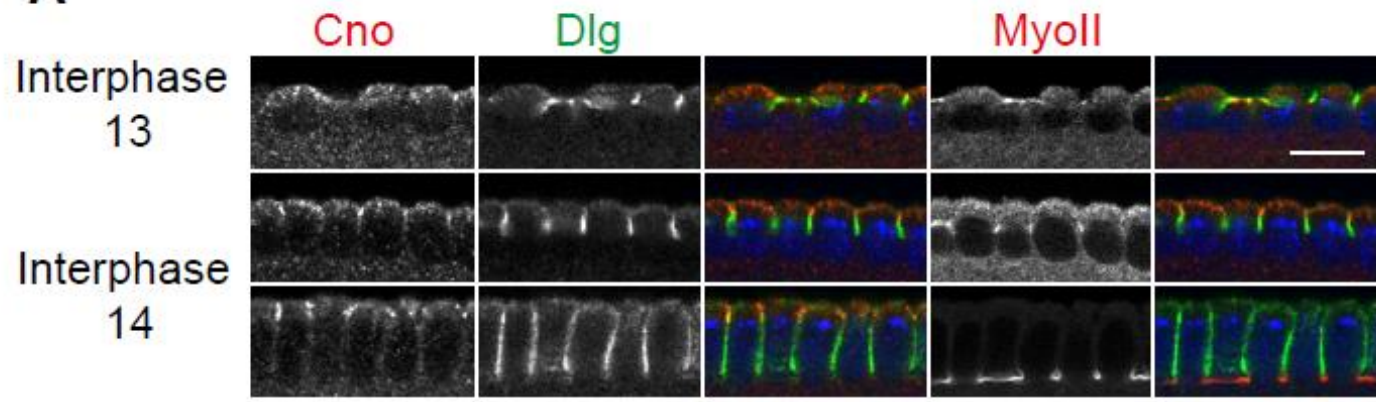

B Mitosis 13

Subapical Basal

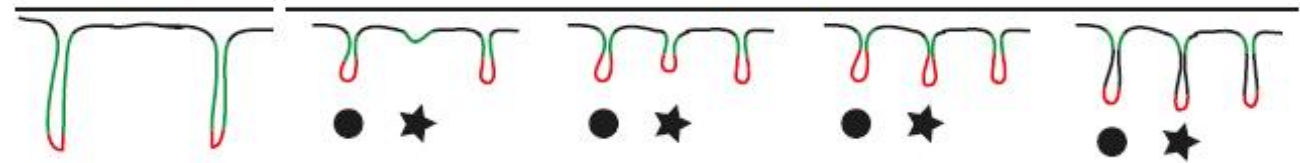

C Mitosis 13

Interphase 14

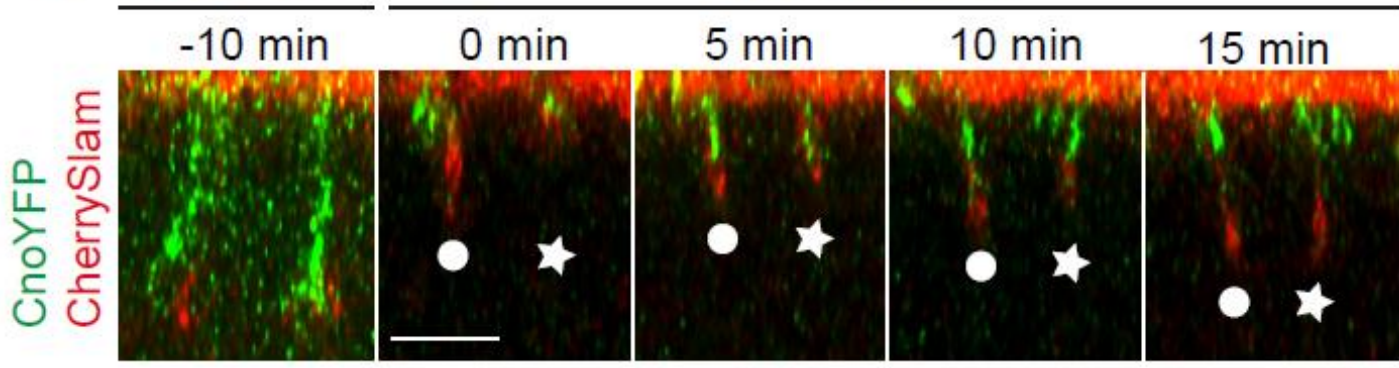

Figure 1 Canoe relocalizes in old cellularizatiation furrows arising from retracting metaphase furrows. (A) Fixed wild type embryos during interphases 13 and 14 as indicated stained against Canoe (grey/red), Dlg (grey/ green), MyosinII (grey/ red) and DNA (blue). (B) Scheme of metaphase and cellularization furrows during mitosis 13 and switch to interphase 14 as indicated. Proteins localizing to the subapical domain during interphase 14 (green) localize to the whole cortex of metaphase furrows. After mitosis 13 metaphase furrows retract and come to a halt forming "old furrows" (circle) while "new furrows" form between (star). The furrwos move inwards synchronosly when they have reached the same length. (C) Living embryos expressing CanoeYFP (green) and CherrySlam (red) to mark subapical and basal domains. Orthogonal views are shown. Stages are as indicated. Scale bar $10 \mu \mathrm{m}$. 
118 Formation of the subapical domain depends on zygotic gene expression but not

\section{9 the nucleo-cytoplasmic ratio}

120 The change from syncytial to cellular blastoderm at the onset of cell cycle 14 and

121 cellularization requires zygotic gene expression (35). Although it is clear that

122 cellularization depends on zygotic gene expression, its functional relationship to the 123 emergence of the subapical domain has not been investigated. ELMO, Sponge, Rap1

124 and Canoe are maternally derived proteins, whose total levels are assumed not to

125 change much. Rather, their distribution on the plasma membrane is controlled by post-

126 translational mechanisms.

127 We first asked whether the spatial restriction of subapical markers depended on zygotic

128 transcription. We analyzed embryos, in which zygotic transcription was blocked by $\alpha-$

129 amanitin, an efficient inhibitor of RNA polymerase II. Injection of $\alpha$-amanitin impairs

130 furrow invagination and cellularization (35). Early embryos expressing CanoeYFP

131 were injected with $\alpha$-amanitin prior to cellularization (Figure 2A). In fixed control

132 embryos, Sponge and CanoeYFP marked the invaginating furrows in a hexagonal

133 pattern, enclosing the nuclei as visible in surface views (Figure 2A). In contrast, no

134 spatial restriction of Sponge and CanoeYFP was detected in injected embryos in

135 interphase 14 , indicating that the restriction of the subapical markers depends on

136 zygotic transcription (Figure 2B). To better resolve the dynamics and staging of the

137 embryos, we recorded time lapse images of embryos expressing ELMO-GFP (Figure

138 2C-D). Control embryos showed a stereotypic ELMO localization at caps during

139 syncytial blastoderm stages and transition to subapical rings during the first few

140 minutes of cellularization in interphase 14 (Figure 2C). In embryos treated with $\alpha$ -

141 amanitin, the cap staining during syncytial blastoderm stage was comparable to control

142 embryos. In contrast, the ELMO-GFP signal remained widely distributed over the

143 whole cortex without any obvious spatial restriction after mitosis 13 (Figure 2D). This 
bioRxiv preprint doi: https://doi org/101101/713719; this version posted July 24, 2019. The copyright holder for this preprint (which was not certified by peer review) is the author/funder, who has granted bioRxiv a license to display the preprint in perpetuity. It is made available under aCC-BY 4.0 International license.

144 loss of restriction of ELMO-GFP was observed at a time when the morphologically

145 visible furrows has not yet formed in control embryos. Our data indicate that spatial

146 restriction of ELMO, Sponge and Canoe in interphase 14 and thus formation of the

147 subapical domain depends on zygotic transcription.

148
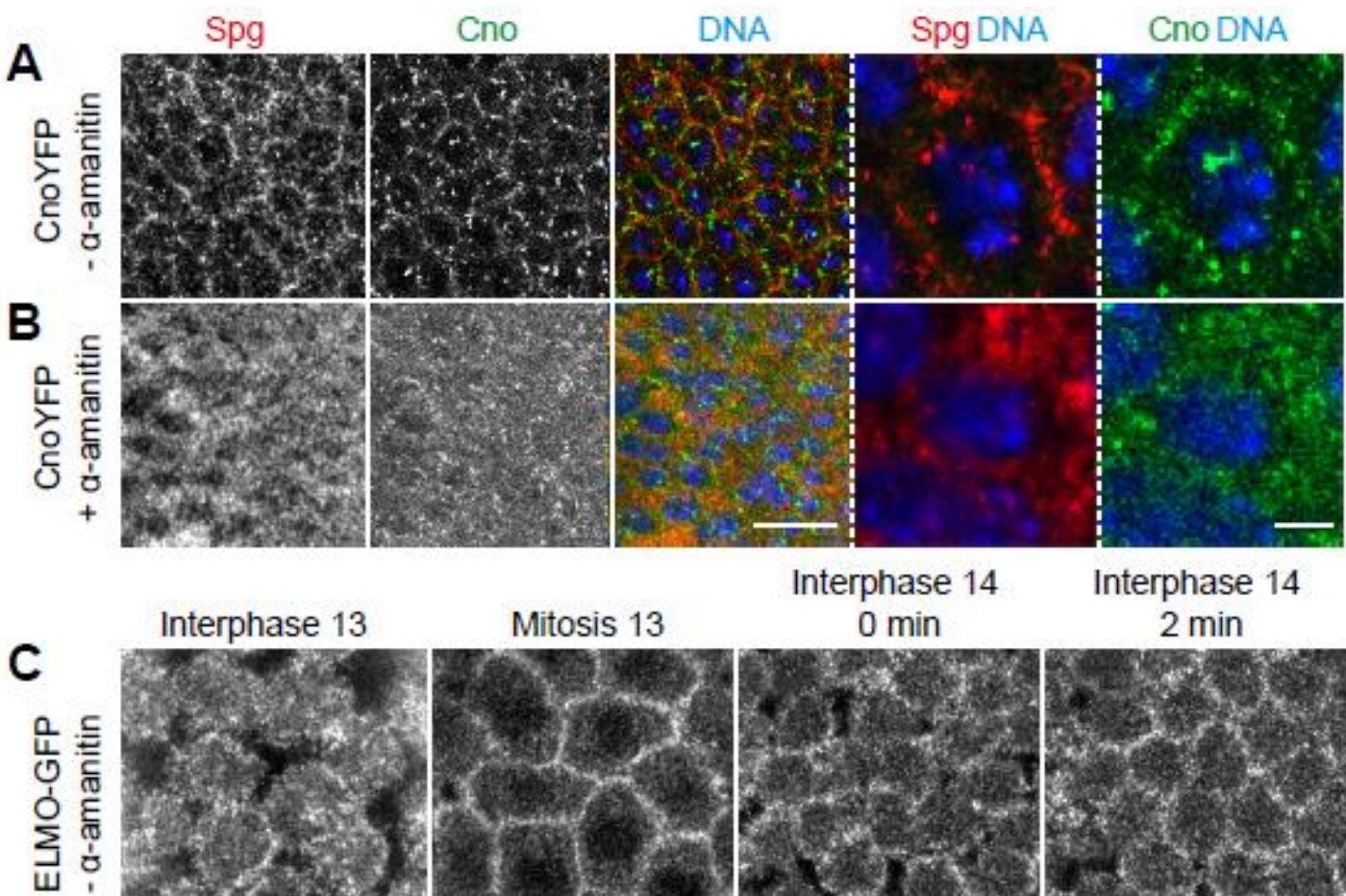

Interphase 13

Mitosis 13

$0 \mathrm{~min}$

2 min
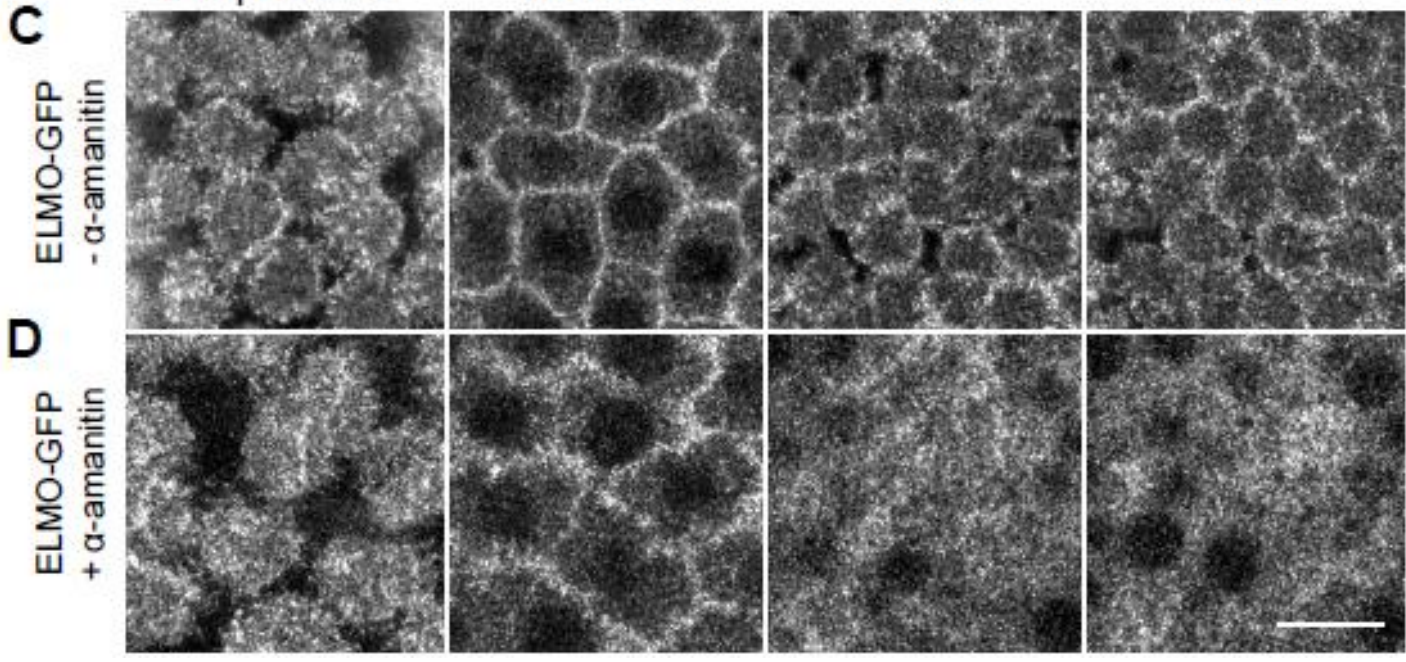

150 Figure 2 Zygotic gene expression is necessary for the formation of the subapical domain during

151 cellularization. (A, B) Fixed non-injected (A) and $\alpha$-amanitin-injected (B) embryos expressing

152 CanoeYFP stained against Sponge (grey/red), CanoeYFP (grey/green) and DNA (grey/ blue)

153 during interphase 14. Merged images and zoom-ins are shown in right panels. (B, D) Top

154 views of images from time lapse movies of non-injected (C) and $\alpha$-amanitin-injected (D) 
$156 \mu \mathrm{m}$.

157 A second obvious timing mechanism beside onset of zygotic transcription during the

158 transition from syncytial to cellular blastoderm is the nucleocytoplasmic ratio. Haploid

159 embryos undergo an extra nuclear division and cellularize only in interphase 15 (36)

160 (Figure 3C, D). It was previously reported that haploid embryos showed features of a

161 cellularization furrow already in interphase 14, i. e. transient basal accumulation of

162 Myo II at the furrow tip (37,38). Cortical domains have otherwise not been specifically

163 investigated in haploid embryos, yet. We fixed and stained haploid embryos from

164 sésame (ssm, Hira) females (21) for Canoe and F-actin. We detected specific subapical

165 restriction of Canoe in cellularizing embryos in interphase 14 as well as in interphase

16615 (Figure $3 \mathrm{C}, \mathrm{D})$. Consistent with the previously reported basal restriction of MyoII,

167 these data suggest that the transient furrow during interphase 14 in haploid embryos

168 contains a patterned cortex with a subapical region. We conclude that the emergence

169 of the subapical and basal domains does not depend on the nucleocytoplasmic ratio.

170 A third timer associated with the midblastula transition is the remodeling of the fast

171 nuclear cycle to a slow cell cycle, which depends on the onset of zygotic transcription

172 (7). We tested whether subapical Canoe restriction would respond to a precocious

173 zygotic transcription and precocious cell cycle remodeling. We analyzed embryos

174 from RPII $215^{X 161}$ germline clones, which precociously start zygotic transcription,

175 cellularize already in interphase 13 , and further develop with half of the number of

176 nuclei (9). By staining of fixed embryos, we detected a normal pattern of F-actin and

177 subapical restriction of Canoe in embryos cellularizing in interphase 13 (Figure 3A,

178 B). 
bioRxiv preprint doi: https://doi org/10.1101/713719; this version posted July 24,2019 . The copyright holder for this preprint (which was not certified by peer review) is the author/funder, who has granted bioRxiv a license to display the preprint in perpetuity. It is made available under aCC-BY 4.0 International license.

179 In summary, our data indicate that the formation of the subapical domain is a regulated

180 feature of the midblastula transition, which responds to zygotic gene expression but

181 not on the nucleocytoplasmic ratio. The failed spatial restriction in embryos with

182 impaired zygotic transcription may be due to the absence of one or multiple specific

183 zygotic factors, which control the distribution pattern of ELMO- Sponge complex, for

184 example. Alternatively, failed spatial restriction may be a consequence of zygotic

185 transcription, such as high polymerase activity or transcription dependent DNA

186 replication stress. Although our time lapse analysis of ELMO-GFP and CanoeYFP

187 indicates that subapical restriction precedes furrow ingression, we do not exclude the

188 possibility that subapical restriction is a consequence of furrow formation due to the

189 limited resolution of our assay.
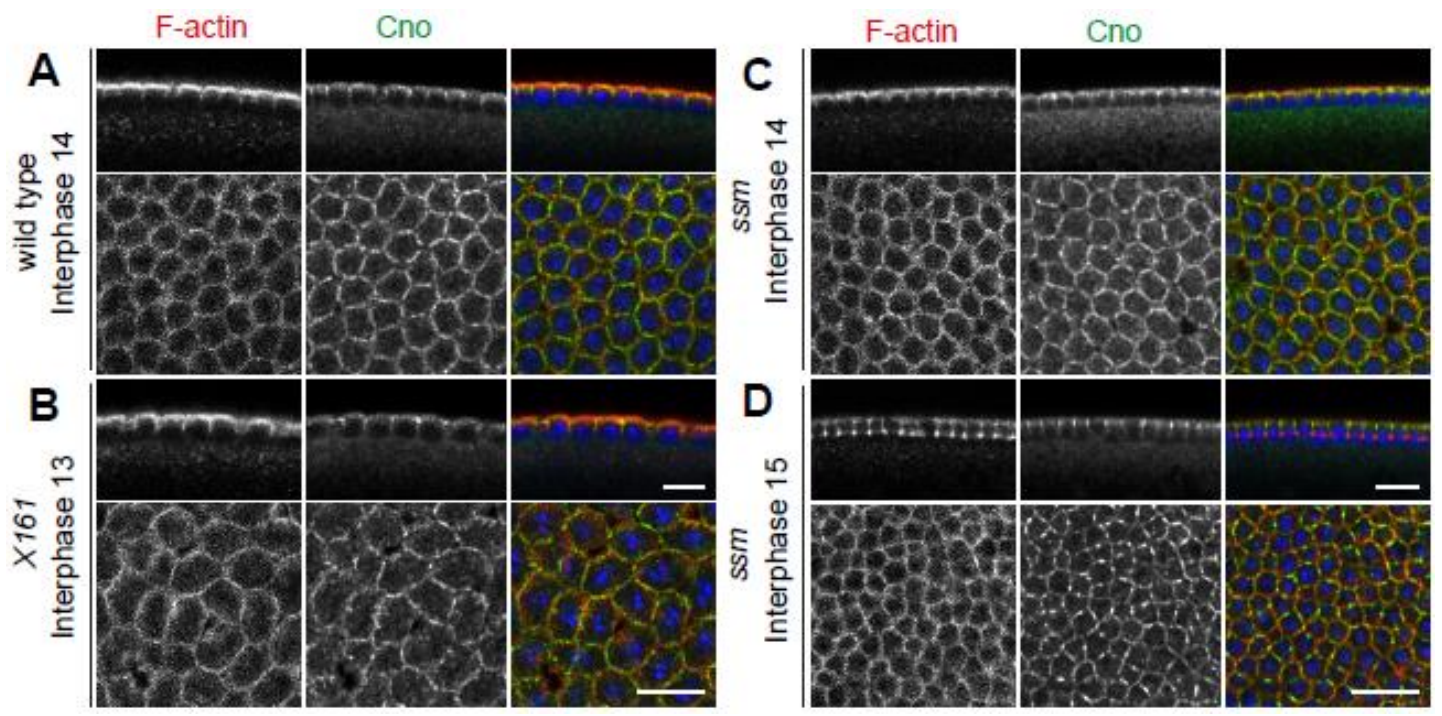

192 Figure 3 Cortical domain formation depends on zygotic gene expression and not on

193 nucleocytoplasmic ratio. (A-D) Fixed wild type (A), X161 (B) and sésame (C, D) embryos

194 stained against F-actin (grey/ red), Canoe (grey/green) and DNA (blue). Merged images are

195 shown in right panels, sagittal sections in top panels and accompanying top views in lower

196 panels. Stages are as indicated. Scale bars $10 \mu \mathrm{m}$. 
197 Bazooka does not regulate subapical Canoe localization during early

198 cellularization

199 Bazooka is a potential zygotic factor controlling subapical restriction of ELMO-

200 Sponge and Canoe, since Bazooka has a maternal and zygotic expression. Previous

201 work revealed a positive feedback mechanism during late cellularization in which

202 subapical restriction of Canoe becomes partially dependent on bazooka (15). We asked

203 whether this feedback interaction was active also during the onset of cellularization.

204 Firstly, we analyzed the distribution of Bazooka and Armadillo which marks E-

205 Cadherin junctions in fixed wild type embryos. For this overview, we imaged all

206 embryos with the same laser settings to compare protein localization and amounts in

207 different stages. With these settings, we did not detect Bazooka at Armadillo positive

208 metaphase furrows during mitosis 13 (Figure 4A). The subapical restriction of

209 Armadillo matures during the course of cellularization starting from an initially wide

210 distribution along the furrow. The basal junction, in comparison, was detected very

211 early on as reported previously (3,39)(Figure 4B-D). A clear subapical Bazooka

212 restriction was first detected during cellularization when the furrows extended to

213 around half the length of the elongated nuclei (Figure 4D). Remarkably, at this time

214 point subapical Armadillo enrichment was not visible yet. During the course of

215 cellularization Bazooka puncta persisted at the subapical position colocalizing with

216 Armadillo $(3,40)$ (Figure 4E, F). The lack of a subapical Bazooka signal during early

217 cellularization does not support an early function of the feedback regulation on Canoe. 


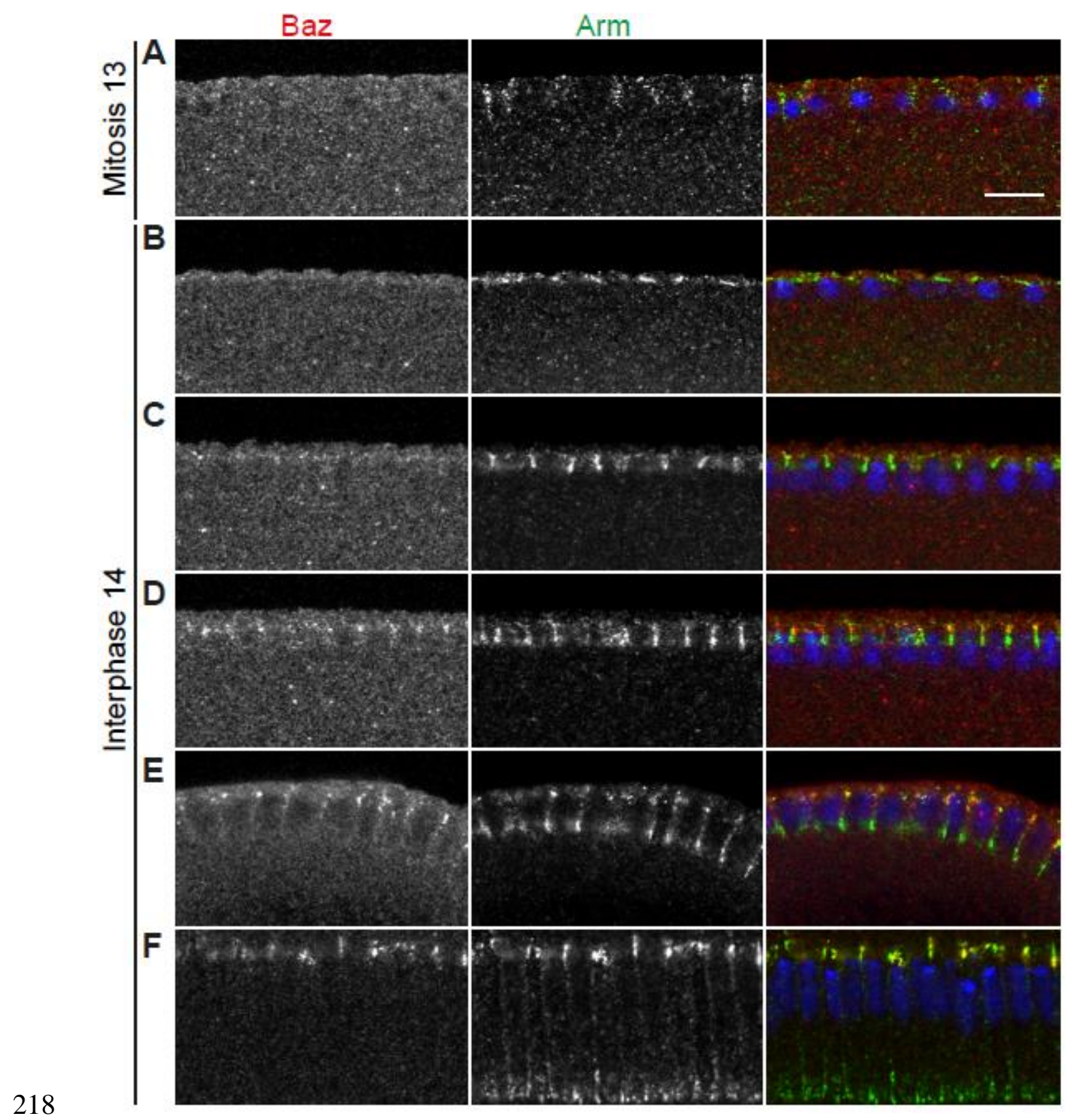

219 Figure 4 Subapical enrichment of Bazooka and Armadillo during cellularization. (A-F) Fixed

220 wild type embryos during mitosis 13 (A) and interphase 14 (B-F, from early to late

221 cellularization). Embryos were stained in the same tube against Bazooka (grey/red), Armadillo

222 (grey/green) and DNA (blue) and imaged with same laser settings to estimate different protein

223 amounts in different stages. Scale bar $10 \mu \mathrm{m}$.

224 To clarify the relation of Canoe and Bazooka in functional terms, we depleted bazooka

225 by RNAi and analyzed fixed and stained embryos (Figure 5). RNAi depletion is

226 functional as indicated by the loss of Bazooka staining and the later phenotype with 
bioRxiv preprint doi: https://doi org/10.1101/713719; this version posted July 24, 2019. The copyright holder for this preprint (which was not certified by peer review) is the author/funder, who has granted bioRxiv a license to display the preprint in perpetuity. It is made available under aCC-BY 4.0 International license.

227 holes in the amnioserosa (Supplemental figure 1). Subapical Canoe enrichment was

228 comparable in wild type controls and bazookaRNAi embryos during early

229 cellularization whereas Canoe localization was affected as described before in early

230 gastrulating embryos (15) (Supplemental figure 1B). Based on these data we conclude

231 that the Bazooka-Canoe feedback loop becomes activated only during the course of

232 cellularization and is not involved in the initial subapical restriction of Canoe.

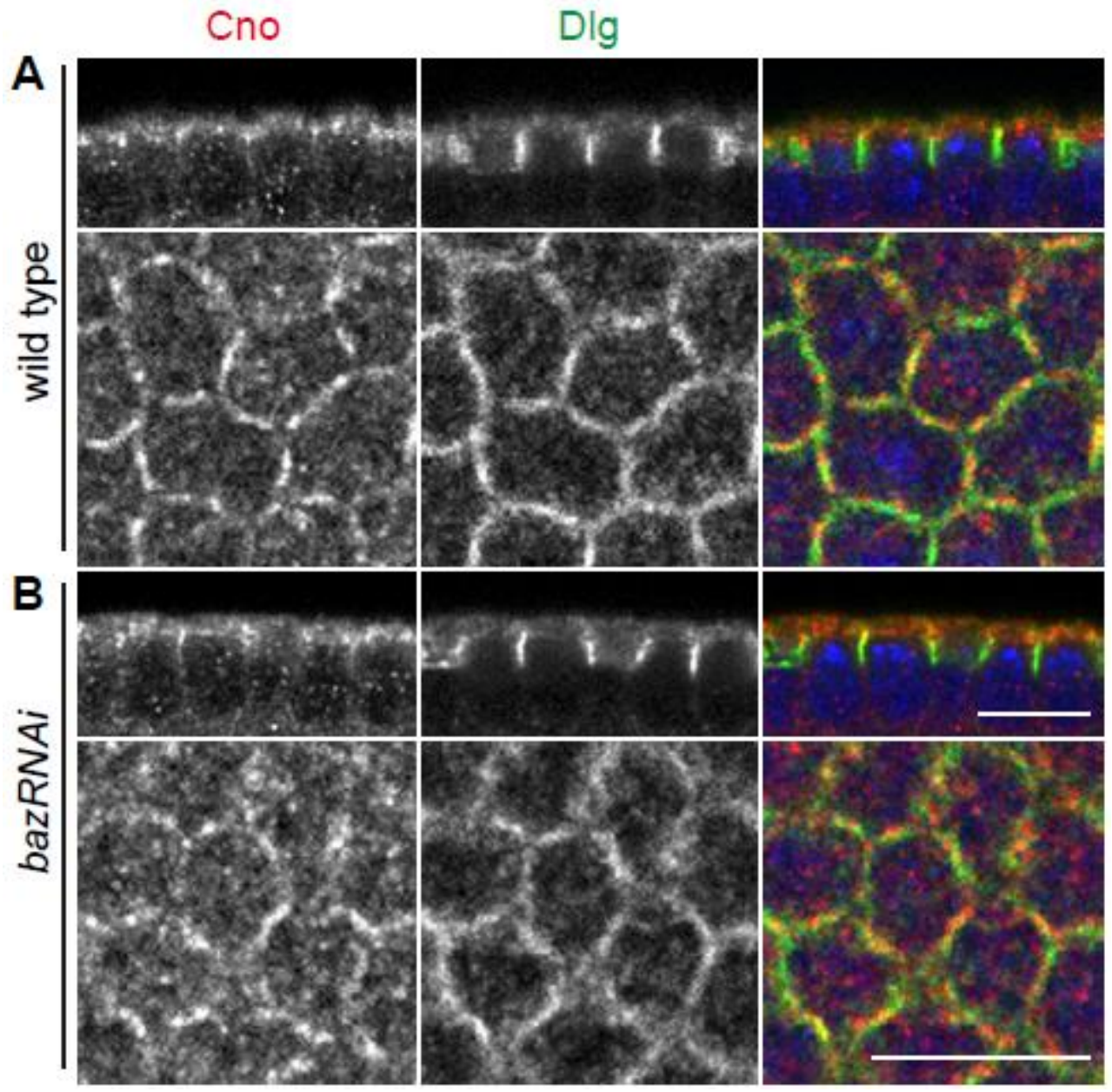

235 Figure 5 Canoe localization is not affected by Bazooka during early cellularization. (A-B)

236 Fixed wild type (A) and bazRNAi (B) embryos during early cellularization stained against

237 Canoe (grey/ red), Dlg (grey/green) and DNA (blue). Side views are shown in upper panels

238 and corresponding top views in lower panels. Scale bars $10 \mu \mathrm{m}$. 
239 Subapical Bazooka enrichment is controlled by the unconventional GEF ELMO-

240 Sponge

241 Initial subapical restriction of Canoe is controlled by the unconventional GEF complex

242 ELMO-Sponge and the GTPase Rap1. We asked whether this functional dependence

243 also holds true for Bazooka and Armadillo. By analysis of fixed embryos, we found

244 that both Rapl and ELMO were required for subapical restriction of both Bazooka and

245 Armadillo. Bazooka and Armadillo staining was dispersed along the lateral furrow in

246 embryos from females with ELMO as well as Rap1 germline clones consistent with

247 previous reports (15) (Figure 6B, D). Conversely Bazooka and Armadillo did not

248 depend on a different Rap1GEF, dizzy, (Figure 6C) consistent with our previous report

249 that subapical restriction of Canoe did not depend on dizzy (8). These findings confirm

250 the earlier described pathway of Bazooka being downstream of the unconventional

251 Rap1 GEF complex ELMO-Sponge, Rap1GTPase and Canoe during early and late 252 cellularization. 


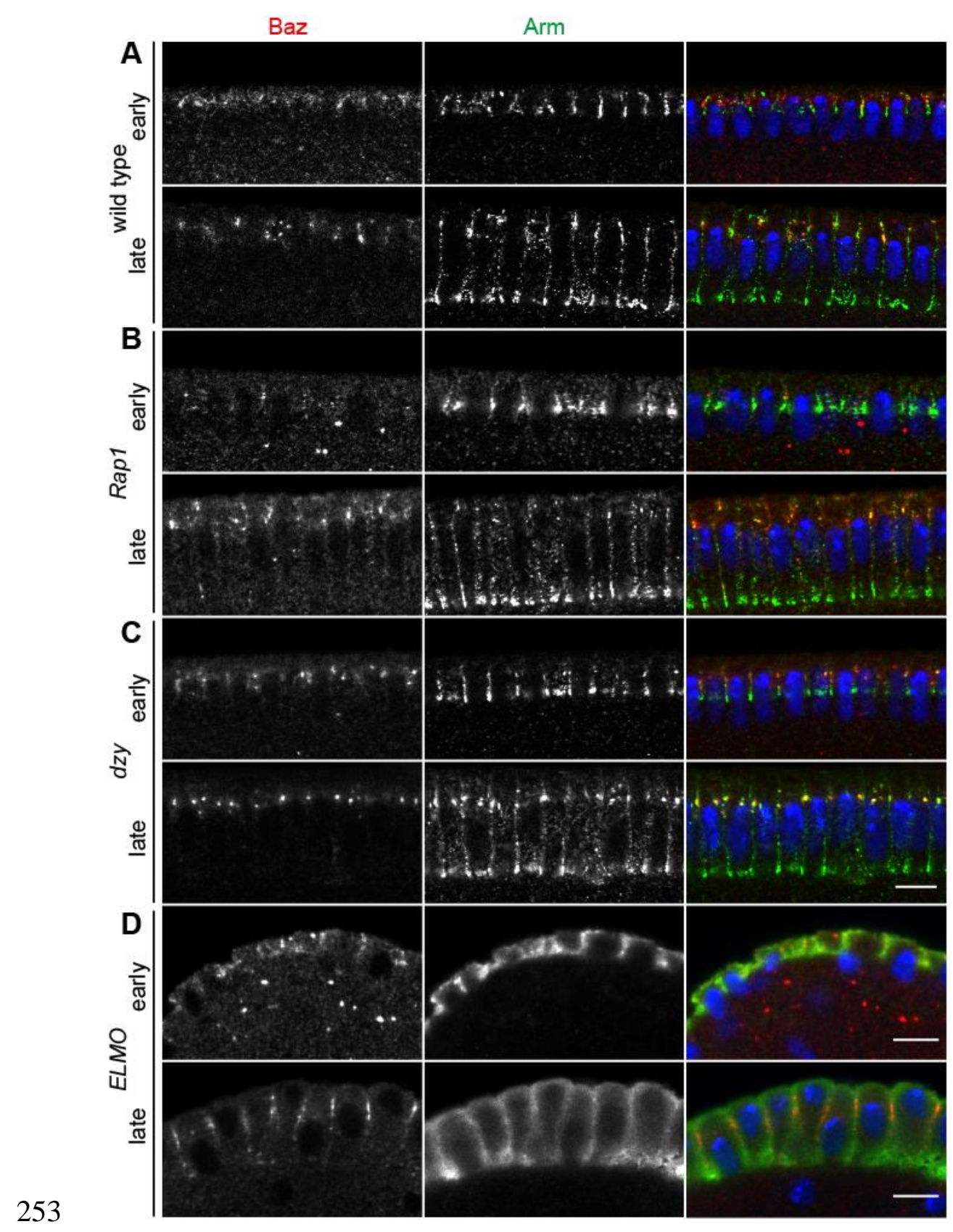

254 Figure 6 Subapical enrichment of Baz and Arm is perturbed in Rap1 and ELMO but not in $d z y$

255 mutants. (A-D) Fixed cellularizing wild type (A), Rapl (B), dzy (C) and ELMO (D) embryos

256 stained against Baz (grey/ red), Arm (grey/ green). DNA is shown in blue. (A) Wild type

257 embryos during early and late cellularization showed subapical Baz and Arm enrichment. (B)

258 Baz puncta are spread along the lateral membrane in early and late cellularization of Rap I

259 embryos. The subapical Arm enrichment was lost whereas basolateral enrichment was still

260 visible. (C) The subapical enrichment of Baz and Arm is not perturbed in early and late 
262 mutants during early and late cellularization. Scale bars $10 \mu \mathrm{m}$.

\section{Discussion}

264 In this study we focused on the function of zygotic gene expression on the formation

265 of the subapical domain during onset of cellularization. Next to the already known

266 fact, that ELMO-Sponge and Canoe localize to newly forming cellularization furrows

267 at onset of interphase 14 (8), we were able to show, that Canoe quickly changes its

268 distribution pattern at "old" cellularization furrows (Figure 7). Although we were able

269 to show Canoe preceding Bazooka to localize to the newly forming furrow and

270 subapical domain, with Bazooka being gradually enriched at the subapical domain

271 during the course of cellularization, we confirmed that Canoe is initially restricted

272 independently of Bazooka. In this analysis we relayed on RNAi mediated depletion,

273 since bazooka has an essential early function in germline determination (41-43).

274 The features of the midblastula transition include deceleration and remodeling of the

275 cell cycle, degradation of maternal products and the switch from syncytial to a cellular

276 blastoderm and the onset of zygotic gene expression $(13,36,44,45)$. As new feature of

277 the morphological changes associated with the midblastula transition we describe here

278 a change in cortical patterning, i. e. the emergence of the subapical domain. Although

279 the restriction of subapical markers precedes formation of a morphologically visible

280 furrow, the apposition of two plasma membranes in initial furrow formation could be

281 the cause of marker restriction, given the limited morphological resolution of our 282 assays. A hint could come from the "old" cellularization furrows that arise from 283 metaphase furrows, which were still detectable in $\alpha$-amanitin injected embryos by

284 ELMO-GFP. Even at the positions of the old furrow the spatial restriction is lost. A 285 limitation to this argument is again the limited insight into the cellular morphology 
286 and dynamics, as the dynamics of the metaphase furrow in embryos lacking zygotic

287 transcription is not clear. A more defined insight into the timing by zygotic gene

288 expression comes from our investigations of embryos with precocious onset of zygotic

289 gene expression. We could detect Canoe at forming cellularization furrows whenever

290 zygotic gene expression was initiated.

291 The next arising question is which zygotic gene or genes could be responsible for

292 relocalization of the subapical domain proteins with onset of cellularization. Among

293 the described early zygotic genes like slam, nullo, bottleneck and serendipity- $\alpha$ no

294 such phenotypes have been described yet $(8,18,46-48)$. However, as general

295 morphology was the primary assay for the screen of zygotic genes $(49,50)$, the

296 subapical determinant might have been missed. A molecular screen of aneuploid

297 embryos for mislocalization of subapical domain proteins may allow the identification

298 of these genes, for example. Although bazooka is already maternally expressed, it

299 seems to take over the function as the subapical determinant only later during in

300 cellularization $(15,16)$. Although, it is not clear how much the expression levels were

301 reduced in bazookaRNAi embryos, we were not able to detect Bazooka protein by

302 staining.

303 Taken together, we were able to show, that the formation of the newly established

304 subapical domain is a novel feature of the midblastula transition, which depends on

305 the onset of zygotic transcription. We propose the hypothesis, that a yet unknown

306 zygotic gene triggers the signaling cascade for subapical domain formation involving

307 ELMO-Sponge, Rap1 and Canoe. 


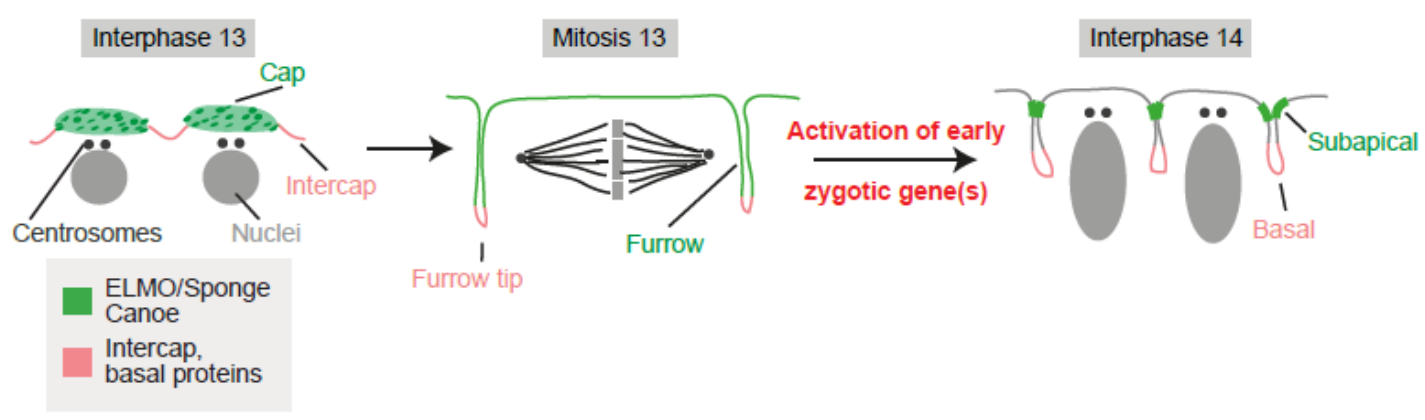

309 Figure 7 Model of cortical domain protein dynamics from interphase 13 to interphase 14.

310 During interphase 13 cortical domain proteins divide in cap (green) and intercap (red)

311 localization, later subapical proteins like Canoe localize to the whole metaphase furrow during

312 mitosis 13 (green), whereas basal proteins (red) localize to the tip and stay there with

313 remodeling to cellularization furrows. With midblastula transition and onset of zygotic gene

314 expression a yet unknown zygotic gene leads to the remodeling of future subapical proteins in

315 old and new cellularization furrows and subapical domain formation is initiated.

\section{Materials and Methods}

\section{Fly stocks and handling}

318 Fly stocks used were CanoeYFP (cno[CPTI000590], Drosophila Genomics and

319 Genetic Resources, Kyoto), UASp-CherrySlam driven by maternal Gal4 (18),

320 rapl[P5709] (R. Reuter, University of Tübingen, Germany)(19) , dizzy(48) (R.

321 Reuter) (20), RPII215[X161] (9), sésame (Hira[185b]) (21); UASbazRNAi

322 (Bloomington stock \# 35002), maternal triple driver MTD-Gal4 (22). As wild type

323 control $w[1118]$ was used.

324 All fly stocks were provided by the Drosophila Stock Center, Bloomington, if not

325 stated differently. Genetic markers and annotations are described in Flybase

326 (http://flybase.org)(23). All crosses and cages were kept at $25^{\circ} \mathrm{C}$. Germ line clones

327 were produced with the ovo/Flipase technique as described previously (24). bazooka 
328 was depleted by overexpression of a short hairpin RNA with MTD-Gal4 during

329 oogenesis.

\section{Immunostainings and antibodies}

331 Following primary antibodies were employed: mouse anti-Armadillo (1:50; N27A1,

332 Hybridoma Center); rabbit anti-Bazooka (1:1000; A. Wodarz)(25); rabbit anti-Canoe

333 (1:1000; (15)); mouse anti-Dlg (1:100; 4F3, Hybridoma Center); guinea pig anti-

334 Sponge $(1: 1000 ;(26))$. F-actin was stained by Phalloidin coupled to Alexa647

335 (Thermo Fisher). Secondary antibodies were labeled with Alexa 488, 568, 647

336 (Thermo Fisher). GFP tagged proteins was detected with GFP-booster coupled with

337 Atto488 (1:500; Chromotek). DNA was stained by DAPI $(0.2 \mu \mathrm{g} / \mathrm{ml}$; Thermo Fisher $)$.

338 Embryos were fixed by $4 \%$ formaldehyde or by heat fixation using standard methods

339 described previously (27) and stored in methanol at $-20^{\circ} \mathrm{C}$. For F-actin staining with

340 phalloidin and in the $\alpha$-amanitin experiments, embryos were fixed by $8 \%$

341 formaldehyde and manually released from the vitelline membrane. For staining,

342 embryos were transferred to PBT (Phosphate buffered saline (PBS) + 0.2\% Tween20),

343 washed trice for $5 \mathrm{~min}$ and afterwards blocked for 30-60 min in PBT+5\% bovine

344 serum (BSA). Embryos were incubated with primary antibodies in PBT+0.1\% BSA

345 overnight at $4^{\circ} \mathrm{C}$ or for $2-3 \mathrm{~h}$ at room temperature. Afterwards the embryos were

346 washed with PBT trice for 15 min, incubated with secondary antibodies in PBT for 1-

$3472 \mathrm{~h}$ at room temperature and again washed $3 \times$ with PBT for $15 \mathrm{~min}$ and stained with

348 DAPI for 10 min at room temperature. The embryos were mounted in Aquapolymount

349 (Thermo Fisher).

350 Injection of $\alpha$-amanitin for inhibition of RNA polymerase II was conducted with a

351 concentration of $1 \mathrm{mg} / \mathrm{ml}$ in water according to standard procedures as described

352 before $(28,29)$. Afterwards, the embryos were staged to reach interphase $14 / 15$ and 
353 fixed as described above. The vitelline membrane was manually removed prior to the

354 staining procedure.

\section{Imaging and Software}

356 Imaging was performed with a Zeiss LSM780 confocal microscope equipped with an

357 Airyscan detector unit. Fixed samples were imaged with an LCI Plan Neofluar

$35863 \times /$ water NA 1.3 objective. Live imaging was conducted with a Plan Neofluar $63 \times /$ oil

359 NA 1.4 objective. Embryos for live imaging were prepared as previously described

360 (30). Fixed samples were imaged with a frame size of $512 \times 512$ pixel $(67.5 \times 67.5 \mu \mathrm{m}$;

$361130 \mathrm{~nm}$ lateral pixel size) for top views and 512×200 pixel $(96.4 \times 29.4 \mu \mathrm{m} ; 190 \mathrm{~nm}$

362 lateral pixel size) for side views. Top views were conducted as z-stacks with a step

363 size of $0.5 \mu \mathrm{m}$. Live imaging was conducted in the Airyscan mode with a frame size

364 of $376 \times 376$ pixel $(31.7 \times 31.7 \mu \mathrm{m}, 80 \mathrm{~nm}$ lateral pixel size $)$. Top views were conducted

365 as axial stacks with a step size of $0.25 \mu \mathrm{m}$. Orthogonal views were constructed in

366 Fiji/ImageJ (31). Image were processed in Fiji/ ImageJ, Adobe Photoshop and

367 Illustrator.

\section{Acknowledgement}

369 We are grateful to E. Geisbrecht, R. Reuter, M. Peifer and A. Wodarz for materials or

370 discussions. We acknowledge service support from the Developmental Studies

371 Hybridoma Bank created by NICHD of the NIH/USA and maintained by the

372 University of Iowa, the Bloomington Drosophila Stock Center (supported by NIH

373 P40OD018537). This work was in part supported by the Deutsche

374 Forschungsgemeinschaft (DFG SPP1464 GR1945/4-2 and equipment grant

375 INST1525/16-1 FUGG). 


\section{References}

377 1. Honigmann A, Pralle A. Compartmentalization of the Cell Membrane. J Mol 378 Biol. 2016 Dec 4;428(24, Part A):4739-48.

379 2. Bilder D, Schober M, Perrimon N. Integrated activity of PDZ protein complexes 380 regulates epithelial polarity. Nat Cell Biol. 2003 Jan;5(1):53-8.

381 3. Harris TJC, Peifer M. The positioning and segregation of apical cues during 382 epithelial polarity establishment in Drosophila. J Cell Biol. 2005 Aug $29 ; 170(5): 813-23$.

4. Schmidt A, Grosshans J. Dynamics of cortical domains in early Drosophila development. J Cell Sci. 2018 Apr 1;131(7):jcs212795.

5. Foe VE, Alberts BM. Studies of nuclear and cytoplasmic behaviour during the five mitotic cycles that precede gastrulation in Drosophila embryogenesis. J Cell

6. Foe VE, Odell GM, Edgar BA. Mitosis and morphogenesis in the Drosophila embryo: Point and counterpoint. In: The Development of Drosophila melanogaster, M Bate and A Martinez Arias. Cold Spring Harbor Laboratory;

7. Liu B, Grosshans J. Link of Zygotic Genome Activation and Cell Cycle Control. In: Zygotic Genome Activation [Internet]. Humana Press, New York, NY; 2017 [cited 2017 Jul 27]. p. 11-30. (Methods in Molecular Biology). Available from: https://link.springer.com/protocol/10.1007/978-1-4939-6988-3_2 
397 8. Schmidt A, Lv Z, Großhans J. ELMO and Sponge specify subapical restriction of Canoe and formation of the subapical domain in early Drosophila embryos. Development. 2018 Jan 15;145(2):dev157909.

9. $\quad$ Sung H, Spangenberg S, Vogt N, Großhans J. Number of Nuclear Divisions in the Drosophila Blastoderm Controlled by Onset of Zygotic Transcription. Curr

10. Warn RM, Bullard B, Magrath R. Changes in the distribution of cortical myosin during the cellularization of the Drosophila embryo. Development. 1980 Jun

11. Warn RM, Magrath R, Webb S. Distribution of F-actin during cleavage of the Drosophila syncytial blastoderm. J Cell Biol. 1984 Jan;98(1):156-62.

12. Mavrakis M, Rikhy R, Lippincott-Schwartz J. Plasma membrane polarity and compartmentalization are established before cellularization in the fly embryo.

13. Blythe SA, Wieschaus EF. Coordinating Cell Cycle Remodeling with Transcriptional Activation at the Drosophila MBT. Curr Top Dev Biol. 2015 Jan

14. Winkler F, Gummalla M, Künneke L, Lv Z, Zippelius A, Aspelmeier T, et al. Fluctuation Analysis of Centrosomes Reveals a Cortical Function of Kinesin-1. Biophys J. 2015 Sep 1;109(5):856-68.

15. Choi W, Harris NJ, Sumigray KD, Peifer M. Rap1 and Canoe/afadin are essential for establishment of apical-basal polarity in the Drosophila embryo. Mol Biol Cell. 2013 Apr;24(7):945-63. 
420 16. Müller HA, Wieschaus E. armadillo, bazooka, and stardust are critical for early stages in formation of the zonula adherens and maintenance of the polarized blastoderm epithelium in Drosophila. J Cell Biol. 1996 Jul 1;134(1):149-63.

17. Sawyer JK, Harris NJ, Slep KC, Gaul U, Peifer M. The Drosophila afadin homologue Canoe regulates linkage of the actin cytoskeleton to adherens

18. Acharya S, Laupsien P, Wenzl C, Yan S, Großhans J. Function and dynamics of slam in furrow formation in early Drosophila embryo. Dev Biol. 2014 Feb

19. Knox AL, Brown NH. Rap1 GTPase Regulation of Adherens Junction Positioning and Cell Adhesion. Science. 2002 Feb 15;295(5558):1285-8.

20. Huelsmann S, Hepper C, Marchese D, Knöll C, Reuter R. The PDZ-GEF Dizzy regulates cell shape of migrating macrophages via Rap1 and integrins in the

21. Loppin B, Docquier M, Bonneton F, Couble P. The Maternal Effect Mutation sésame Affects the Formation of the Male Pronucleus in Drosophila melanogaster. Dev Biol. 2000 Jun 15;222(2):392-404.

22. Mazzalupo S, Cooley L. Illuminating the role of caspases during Drosophila oogenesis. Cell Death Differ. 2006 Nov;13(11):1950. 8;47(D1):D759-65. 
442 24. Chou TB, Noll E, Perrimon N. Autosomal P[ovoD1] dominant female-sterile

443 insertions in Drosophila and their use in generating germ-line chimeras.

444 Development. 1993 Dec 1;119(4):1359-69.

445 25. Wodarz A, Ramrath A, Kuchinke U, Knust E. Bazooka provides an apical cue 446 for Inscuteable localization in Drosophila neuroblasts. Nature. 1999 $447 \quad$ Dec;402(6761):544.

26. Biersmith B, Liu Z, Bauman K, Geisbrecht ER. The DOCK Protein Sponge Binds to ELMO and Functions in Drosophila Embryonic CNS Development. PLOS ONE. 2011 Jan 25;6(1):e16120.

28. Liu B, Winkler F, Herde M, Witte C-P, Großhans J. A Link between Deoxyribonucleotide Metabolites and Embryonic Cell-Cycle Control. Curr Biol.

29. Yan S, Großhans J. Localization and translation control of slam in Drosophila 2019 Apr 1;29(7):1187-1192.e3.

30. Kanesaki T, Edwards CM, Schwarz US, Grosshans J. Dynamic ordering of nuclei in syncytial embryos: a quantitative analysis of the role of cytoskeletal networks. Integr Biol. 2011 Nov 1;3(11):1112-9. al. Fiji: an open-source platform for biological-image analysis. Nat Methods. 2012 Jul;9(7):676-82. 
465 32. Raff JW, Glover DM. Centrosomes, and not nuclei, initiate pole cell formation in Drosophila embryos. Cell. 1989 May 19;57(4):611-9.

33. He B, Martin A, Wieschaus E. Flow-dependent myosin recruitment during Drosophila cellularization requires zygotic dunk activity. Dev Camb Engl. 2016 Jul 1;143(13):2417-30.

34. Bonello TT, Perez-Vale KZ, Sumigray KD, Peifer M. Rap1 acts via multiple mechanisms to position Canoe and adherens junctions and mediate apical-basal polarity establishment. Development. 2018 Jan 15;145(2):dev157941.

35. Edgar BA, Kiehle CP, Schubiger G. Cell cycle control by the nucleo-cytoplasmic ratio in early Drosophila development. Cell. 1986 Jan 31;44(2):365-72.

36. Lu X, Li JM, Elemento O, Tavazoie S, Wieschaus EF. Coupling of zygotic transcription to mitotic control at the Drosophila mid-blastula transition. Development. 2009 Jun 15;136(12):2101-10.

37. Großhans J, Wenzl C, Herz H-M, Bartoszewski S, Schnorrer F, Vogt N, et al. RhoGEF2 and the formin Dia control the formation of the furrow canal by directed actin assembly during Drosophila cellularisation. Development. 2005 Mar 1;132(5):1009-20.

38. Großhans J, Müller HAJ, Wieschaus E. Control of Cleavage Cycles in Drosophila Embryos by frühstart. Dev Cell. 2003 Aug 1;5(2):285-94. 
487 40. Harris TJC, Peifer M. Adherens junction-dependent and-independent steps in the establishment of epithelial cell polarity in Drosophila. J Cell Biol. 2004 Oct

41. Doerflinger H, Vogt NM, Torres IL, Mirouse V, Koch I, Nüsslein-volhard C, et al. Bazooka is required for polarisation of the Drosophila anterior-posterior axis.

42. Huynh JR, Petronczki M, Knoblich JA, St Johnston D. Bazooka and PAR-6 are required with PAR-1 for the maintenance of oocyte fate in Drosophila. Curr Biol

43. Becalska AN, Gavis ER. Bazooka regulates microtubule organization and spatial restriction of germ plasm assembly in the Drosophila oocyte. Dev Biol. 2010 Apr

44. Sibon OCM, Stevenson VA, Theurkauf WE. DNA-replication checkpoint control at the Drosophila midblastula transition. Nature. 1997 Jul 3;388(6637):93-7.

45. Yuan K, Seller CA, Shermoen AW, O'Farrell PH. Timing the Drosophila Mid-

46. Rose LS, Wieschaus E. The Drosophila cellularization gene nullo produces a blastoderm-specific transcript whose levels respond to the nucleocytoplasmic ratio. Genes Dev. 1992 Jul;6(7):1255-68.

47. Schejter ED, Wieschaus E. bottleneck acts as a regulator of the microfilament network governing cellularization of the Drosophila embryo. Cell. 1993 Oct 
510 48. Schweisguth F, Lepesant JA, Vincent A. The serendipity alpha gene encodes a

511 membrane-associated protein required for the cellularization of the Drosophila

512 embryo. Genes Dev. 1990 Jan 6;4(6):922-31.

513 49. Wieschaus E, Sweeton D. Requirements for X-linked zygotic gene activity 514 during cellularization of early Drosophila embryos. Development. 1988 Nov $515 \quad 1 ; 104(3): 483-93$.

516 50. Merrill PT, Sweeton D, Wieschaus E. Requirements for autosomal gene activity 517 during precellular stages of Drosophila melanogaster. Development. 1988 Nov $518 \quad 1 ; 104(3): 495-509$. 


\section{Supplemental figures}

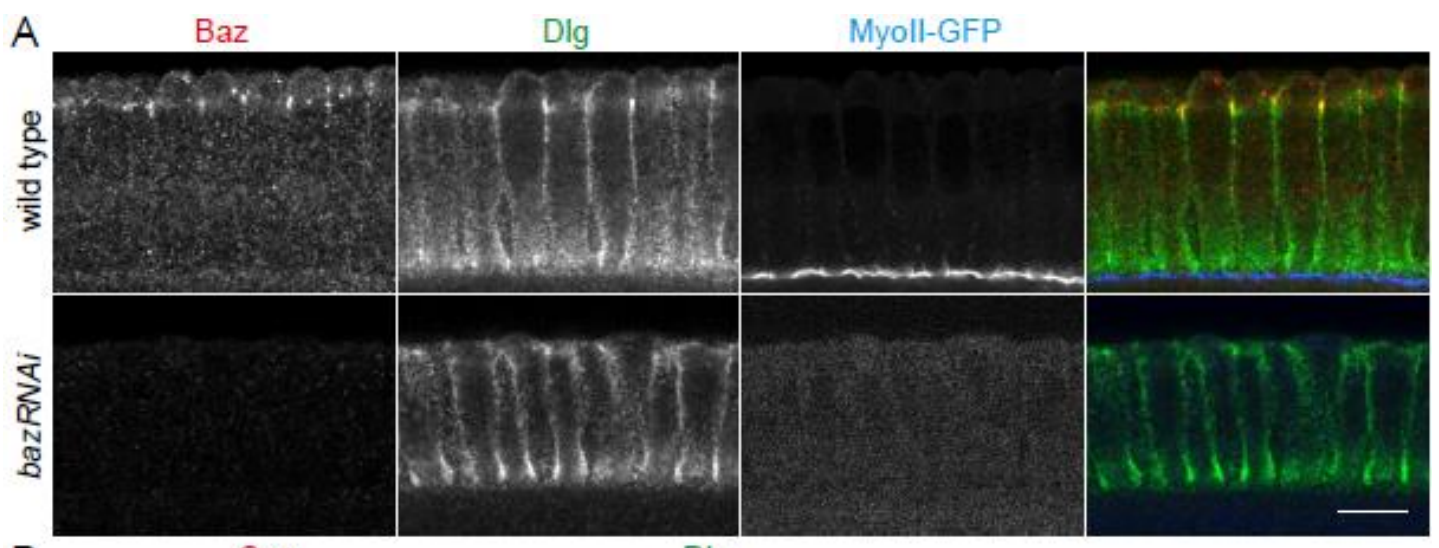

B

Cno

$\mathrm{Dlg}$

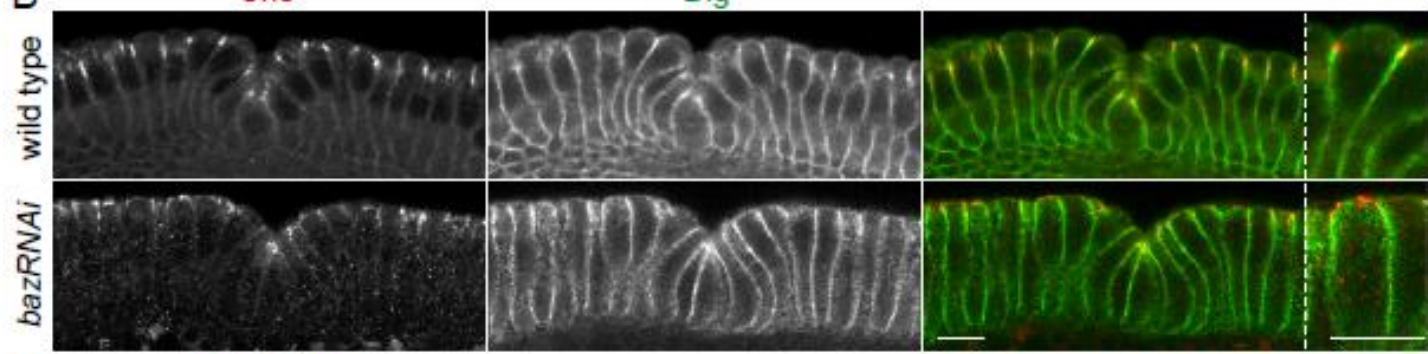

C

Cno DNA

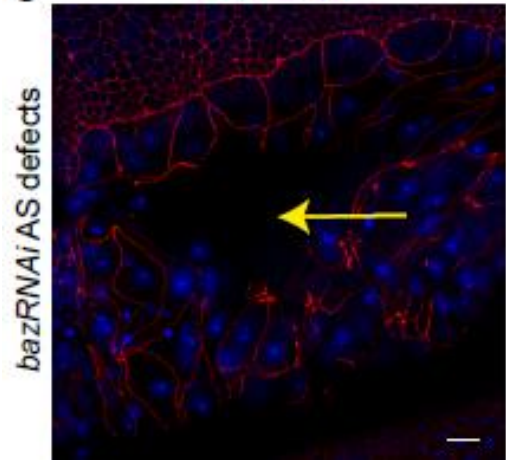

521 Supplemental figure 1 Functionality of bazooka knock down. (A) Wild typic embryos

522 expressing MyoII-GFP and bazRNAi embryos fixed and stained during late cellularization

523 agains Baz (grey/red), Dlg (grey/ green) and MyoGFP (grey/ blue). MyoGFP and bazRNAi

524 embryos were fixed and stained in the same tubes and imaged with same settings. (B) Fixed

525 wild type and bazRNAi embryos during early gastrulation stained against Cno (grey/ red) and

526 Dlg (grey/ green). (C) Stage 13 bazRNAi embryo fixed and stained against Cno (red) and

527 DNA (blue) showing typical amnioserosa holes (yellow arrow). Scale bars $10 \mu \mathrm{m}$. 